Programa de Pós-Graduação em Engenharia de Produção - PPGEP
Laboratório de Qualidade de Vida - LaQVida
Universidade Tecnológica Federal do Paraná - UTFPR
Ponta Grossa - PR - Brasil

DOI: $10.3895 / \mathrm{S} 2175-08582014000100005$

\title{
Correlação da força muscular respiratória com a qualidade de vida e capacidade funcional de idosos institucionalizados
}

\section{Correlation of respiratory muscle strength with quality of life and functional capabilities of institutionalized elderly}

\author{
Adriana Dutra Gomes \\ Faculdade Anglo-Americano - FAA - Foz do Iguaçu - Paraná - Brasil \\ dinanana@gmail.com \\ Jocimar Ferreira Silva \\ Faculdade Anglo-Americano - FAA - Foz do Iguaçu - Paraná - Brasil \\ jocimarsilva@yahoo.com.br \\ Juliana Aleixo da Silva \\ Faculdade Anglo-Americano - FAA - Foz do Iguaçu - Paraná - Brasil \\ julianaaleixodasilva@hotmail.com \\ Andersom Ricardo Fréz \\ Universidade Estadual do Centro-Oeste - UNICENTRO - Guarapuava - Paraná - Brasil \\ andersom_frez@yahoo.com.br \\ Cintia Teixeira Rossato Mora \\ Faculdade Anglo-Americano - FAA - Foz do Iguaçu - Paraná - Brasil \\ cintiatr_mora@hotmail.com \\ Christiane Riedi \\ Universidade Estadual do Centro-Oeste - UNICENTRO - Guarapuava - Paraná - Brasil \\ chrikets@gmail.com
}

\section{RESUMO}

OBJETIVO: Verificar se existe correlação entre força muscular respiratória e qualidade de vida e capacidade funcional de idosos institucionalizados.

MÉTODOS: Foi realizado um estudo transversal com 16 idosos institucionalizados. Para avaliar a qualidade de vida foi aplicada a versão abreviada do questionário WHOQOL e para mensurar a capacidade funcional foram aplicados a Escala de Katz e o teste de caminhada de seis minutos. As forças musculares respiratórias foram avaliadas utilizando um manovacuômetro analógico. A qualidade de vida e a capacidade funcional foram correlacionadas com a força muscular respiratória, utilizando o teste de correlação não paramétrica de Spearman.

RESULTADOS: A amostra estudada apresentava forças musculares respiratórias e distância percorrida no teste de caminhada de seis minutos abaixo das médias preditas para a faixa etária ( $\mathrm{p}<0,0001)$. Em relação ao grau de dependência/independência, a amostra caracterizou-se como independente. Não foram observadas correlações entre as variáveis analisadas $(p>0,05)$.

CONCLUSÃO: Não foram observadas correlações entre a força muscular inspiratória e expiratória com a capacidade funcional e qualidade de vida em idosos institucionalizados. 
PALAVRAS-CHAVE: Força muscular. Avaliação em saúde. Avaliação geriátrica. Instituição de longa permanência para idosos.

\begin{abstract}
OBJECTIVE: To investigate whether there is a correlation between respiratory muscle strength and quality of life and functional capacity of institutionalized elderly.

METHODS: We conducted a cross-sectional study with 16 institutionalized elderly. To assess the quality of life was applied WHOQOL-bref; and Katz Index and six minute walking test measured the functional capacity. The respiratory muscle strength was assessed using an analog manometer. Quality of life and functional capacity were correlated with respiratory muscle strength using the Spearman test.
\end{abstract}

RESULTS: The sample had respiratory muscle strength and distance walked during the six minute walk test below the predicted averages $(p<0.0001)$. In relation to degree of dependence or independence, the sample was characterized as independent. There were no correlation between the variables $(\mathrm{p}>0.05)$.

CONCLUSION: There were no correlations between respiratory muscle strength and quality of life and functional capacity of institutionalized elderly.

KEYWORDS: Muscle strength. Health evaluation. Geriatric assessment. Homes for the aged.

\title{
1. Introdução
}

O sistema respiratório do idoso sofre progressiva diminuição de seu desempenho em decorrência de alterações estruturais e funcionais. Entre as estruturais, observam-se modificações que ocorrem nos pulmões, na caixa torácica, no drive e musculatura respiratória. Já entre as fisiológicas, ocorre uma redução da complacência da caixa torácica (BRITTO et al., 2005), a qual vem acompanhada da fraqueza dos músculos respiratórios, prejudicando a efetividade da tosse, predispondo a retenção de secreções e o desenvolvimento de infecções brônquio pulmonares. Assim, o idoso torna-se cada vez mais limitado na sua capacidade de desempenhar as atividades da vida diária (PETTENON et al., 2008). Essa limitação produz adversidades que vão desde dificuldades na realização de serviços formais e informais em casa até hospitalizações e admissões em asilos (CADER et al. 2006).

As perdas relacionadas ao envelhecimento quando associadas à doenças crônicas e à inatividade física podem produzir dependência parcial, total ou até mesmo à morte. Desta forma, é muito comum o idoso passar a ser dependente de cuidadores ou familiares para a realização de tarefas do dia a dia. Essa perda da autonomia faz com que os níveis de qualidade de vida apresentem declínio (MINCATO; FREITAS, 2007).

Nesse sentido, avaliar a função muscular respiratória de idosos e suas relações com a capacidade funcional e qualidade de vida pode colaborar para a identificação de medidas terapêuticas mais efetivas (SIMÕES et al., 2010; VASCONCELOS et al., 2007). O objetivo desse estudo foi verificar se existe correlação entre a força muscular respiratória e a qualidade de vida e capacidade funcional de idosos institucionalizados.

\section{Métodos}

Trata-se de um estudo transversal descritivo, aprovado pelo Comitê de Ética e Pesquisa da Faculdade Assis Gurgacz (CEP/FAG), parecer 115/2011. O estudo foi realizado na Associação de Amparo aos Idosos - Lar dos Velhinhos, de Foz do Iguaçu, Paraná. Foram incluídos idosos entre 60 
a 75 anos, tabagistas e ex-tabagistas, sedentários, que tivessem capacidade de deambulação sem assistência, com índice de massa corporal (IMC) caracterizada como normal ou sobrepeso e escore igual ou maior que 24 no Miniexame do Estado Mental (MEEM), abaixo desse valor foi considerado índice de déficit cognitivo. Foram excluídos os portadores de distúrbios ortopédicos ou demais doenças que interferissem na capacidade da marcha, como: fraturas, assimetria de membros inferiores, amputações de membros inferiores, pé torto e pneumopatas com diagnóstico clínico.

Após anuência da responsável da associação, os 43 idosos institucionalizados foram avaliados por um único pesquisador, sem vínculo com a associação. Nessa primeira avaliação foi realizada uma anamnese e a aplicação do MEEM (PAVAN et al., 2010).

A amostra foi composta por 16 idosos, dos quais foram avaliados a qualidade de vida, o grau de dependência, capacidade funcional e forças musculares respiratórias.

A versão abreviada do questionário WHOQOL foi utilizada para avaliar a qualidade de vida. Tratase de um questionário composto de 26 questões extraídas do WHOQOL-100, sendo duas questões gerais sobre a qualidade de vida e as demais distribuídas em quatro domínios: físico, psicológico, relação social e meio ambiente. Quanto mais alto os escores, melhor é a qualidade de vida (FLECK et al., 2000).

O grau de dependência funcional foi avaliado pela Escala de Katz de Independência em Atividades de Vida Diária, traduzida e adaptada transculturalmente para a língua portuguesa (LINO et al., 2008). Essa escala permitiu avaliar a dificuldade do idoso em realizar suas tarefas do dia a dia relacionadas com o cuidado corporal, tais como: vestir-se, banhar-se, transferir-se, usar o banheiro, controlar esfíncter e alimentar-se. Para a pontuação do questionário foi utilizado o formato Likert, no qual indivíduo independente recebeu a pontuação 0 ; se necessita de alguma ajuda material, 1; se precisa de ajuda humana, 2; e o dependente total recebeu pontuação 3, ou seja, a pontuação máxima são 21 pontos, e quanto maior a pontuação maior a dependência.

Também foi realizado o teste de caminhada de seis minutos (TC6). No teste os idosos foram orientados a percorrer o maior número de vezes um trecho demarcado no solo com fitas coloridas e com cones em ambas as extremidades. Foi ressaltado que, caso encontrassem necessidade de diminuir o ritmo ou mesmo de interromper o teste, deveriam fazê-lo. Próximo ao ponto de partida, as variáveis pressão arterial (PA), saturação periférica de oxigênio $\left(\mathrm{SPO}_{2}\right)$ e frequência cardíaca (FC) foram verificados com os sujeitos em repouso. Durante todo o TC6, os sujeitos foram monitorados por meio da escala de Borg e as demais variáveis avaliadas a cada dois minutos. Durante todo o percurso, um examinador acompanhou os sujeitos, posicionando-se póstero-lateralmente, sustentando o oxímetro de pulso para monitorização das variáveis e para segurança dos sujeitos, além de realizar incentivos verbais padronizados pela American Thoracic Society (2002).

As forças musculares respiratórias (PImáx e PEmáx) foram realizadas com manovacuômetro analógico (Medical Clinic). O aparelho foi conectado a uma traquéia de plástico e a um bocal de plástico rígido. Foram realizadas três manobras para o aprendizado, e em seguida três novas medidas, as quais foram consideradas, com intervalo de um minuto entre as medidas. Foi escolhido o maior valor sustentado por um segundo, essas medidas foram realizadas com os voluntários sentados em cadeiras com encosto alto e vestindo roupas confortáveis. Os voluntários usaram clipe nasal, seguraram o aparelho e foram instruídos a colocar os lábios no bocal e mantê-los firmemente para evitar perdas de ar. Para a PImáx os voluntários expiraram no bocal até alcançar o volume residual. Para a PEmáx os voluntários inspiraram no bocal até a capacidade pulmonar total. Durante essas medidas o examinador segurou a musculatura da face (perioral) para evitar o uso da musculatura e também o escape de ar (VASCONCELOS et al., 2007). Após isso, os valores obtidos foram comparados aos previstos pela equação de Neder et al. (1999).

As variáveis quantitativas foram apresentadas em média e desvio-padrões (DP) ou média e valores mínimos e máximos. Já para as variáveis qualitativas foram calculadas as frequências absolutas e relativas. Para comparação das medidas encontradas com as preditas foi aplicado o teste t para dados não pareados, e para correlação entre a força muscular respiratória e a capacidade funcional foi utilizado o coeficiente de correlação de Spearman, com nível de significância estabelecido em 5\%. Os testes foram realizados com o uso do software Graphpad Instat versão 3.0. 


\section{Resultados}

A Tabela 1 apresenta as características da amostra:

\begin{tabular}{lc}
\multicolumn{2}{c}{ Tabela 1 - Caracterização da amostra } \\
\hline Masculino (\%) & 68,8 \\
Feminino (\%) & 31,2 \\
Idade (anos), média (DP) & $67,6(4,3)$ \\
Estatura (centímetros), média (DP) & $165,0(9,0)$ \\
Massa corporal (quilos), média (DP) & $63,8(13,9)$ \\
IMC (Kg/m²), média (DP) & $23,1(4,2)$ \\
MEEM (escore), média (DP) & $29,5(2,5)$ \\
\hline Fonte: Autoria própria (2012).
\end{tabular}

Os valores das forças musculares respiratórias (PImáx e PEmáx) e a distância percorrida no TC6 ficaram abaixo das médias preditas. Em relação ao grau de dependência/independência, observou-se uma amostra de idosos caracterizada como independente (Tabela 2).

Tabela 2 - Resultados dos testes realizados

\begin{tabular}{lccccc}
\hline \multicolumn{1}{c}{ Variáveis } & Valor encontrado & $\begin{array}{c}\text { Valor previsto, } \\
\text { Média (DP) }\end{array}$ & \% previsto & Valor Máximo & Valor p \\
\hline PImáx & $58,3(15,8)$ & $94,0(13,4)$ & $62,1(12,5)$ & - & $<0,0001$ \\
PEmáx & $53,9(21,4)$ & $99,7(19,0)$ & $55(20,8)$ & - & $<0,0001$ \\
TC6 & $336,0(96,0)$ & $546,5(103,4)$ & $63(19)$ & - & $<0,0001$ \\
Escala de Katz (escore) & $5,9(0,3)$ & - & - & 21 & - \\
WHOQoL (escore) & & - & - & 35 & - \\
$\quad$ Dominio Físico & $21,9(4,3)$ & - & - & 30 & - \\
Domínio psicológico & $10,9(3,8)$ & - & - & 15 & - \\
Domínio social & $8,0(1,3)$ & - & - & 40 & - \\
Domínio ambiental & $26,8(4,3)$ & - & - & - \\
\hline
\end{tabular}

Fonte: Autoria própria (2012).

Não foi observada correlação entre a força muscular respiratória e capacidade funcional e qualidade de vida (Tabela 3).

Tabela 3 - Correlação entre a força muscular respiratória e a capacidade funcional

\begin{tabular}{lcccccc}
\hline & \multicolumn{2}{c}{ TC6 } & \multicolumn{2}{c}{ Escala de Katz } & \multicolumn{2}{c}{ WHOQOL } \\
\cline { 2 - 7 } & $\mathrm{R}$ & Valor $\mathrm{p}$ & $\mathrm{R}$ & Valor $\mathrm{p}$ & $\mathrm{R}$ & Valor $\mathrm{p}$ \\
\hline PImáx & $-0,1184$ & 0,6624 & 0,0417 & 0,8779 & 0,1753 & 0,5161 \\
PEmáx & 0,4095 & 0,1152 & $-0,0827$ & 0,7607 & 0,2290 & 0,3935 \\
\hline
\end{tabular}

Fonte: Autoria própria (2012).

\section{Discussão}

Durante as últimas décadas a proporção de idosos aumentou consideravelmente na maioria dos países. As pessoas atualmente vivem durante mais tempo, mesmo sofrendo de doenças crônicas e de limitações funcionais por um período de tempo longo. Assim, torna-se importante identificar fatores que possam ajudar na transição da saúde, criando estratégias preventivas que proporcionem uma maior independência (MOTA et al., 2006). Entretanto, o estilo de vida sedentário, combinado com o processo de envelhecimento, pode agravar a redução da força muscular inspiratória e expiratória, resultando na redução da pressão intratorácica e no fluxo expiratório durante a tosse (FREITAS et al., 2010).

Em uma pesquisa com 4.443 idosos, para determinar valores de referência para PImáx e PEmáx, foram demonstradas correlações positivas com: gênero masculino, força de preensão palmar e maior massa corporal magra; e correlações negativas para: idade avançada, baixa estatura e 
tabagismo (ENRIGHT et al., 1994). Assim, espera-se que os homens tenham melhor desempenho funcional que as mulheres (SIMÕES et al., 2010). Entretanto, na presente pesquisa essa correlação não foi avaliada. Foram consideradas as correlações entre PImáx e PEmáx e a qualidade de vida, grau de dependência e capacidade funcional, porém, não foram observadas correlações. Sobre a manovacuometria, uma técnica rápida, simples e não invasiva e com reprodutibilidade aceitável, não foram observadas limitações na sua aplicação, corroborando com os achados de Vasconcelos et al. (2007).

Para avaliar a capacidade funcional foram aplicados o TC6 e a Escala de Katz. O TC6 foi introduzido inicialmente na década de 70 e validado como uma adaptação ao teste de corrida de 12 minutos (teste de Cooper). É um teste de fácil aplicação, interpretação, não oneroso, rápido, simples e bem tolerado pelos pacientes. Para o idoso é um teste vantajoso, pois ele escolhe sua própria velocidade e permite lhe descansar durante a execução, caso se canse ou sinta algum desconforto (ARAUJO et al., 2006). Na presente pesquisa, o TC6 demonstrou-se como um teste bem tolerado por todos os idosos, pois não houve variações que pudessem trazer risco para eles. Caracterizando-se, assim, como um método de avaliação seguro, pois o esforço que o idoso precisa realizar é pequeno, sendo um teste submáximo (DOURADO, 2011). Apesar do TC6 ter sido bem aceito e todos os idosos terem conseguido concluir o percurso sem apresentar fadiga constante, ficou evidente a diferença entre a média caminhada com a predita, demonstrando um comprometimento funcional nos idosos do estudo. Acredita-se que este comprometimento possa estar relacionado à institucionalização, pois idosos comunitários conseguem caminhar as distâncias previstas pelo TC6 (ANJOS et al., 2012). Além disso, quanto maior o grau de independência, melhor a aptidão física, a qual contribui para a manutenção da capacidade funcional (MAZO et al., 2011).

Esperava-se que entre idosos institucionalizados o comprometimento funcional pudesse ser correlacionado com uma possível redução da força muscular respiratória. Mesmo a avaliação da força muscular respiratória e do TC6 terem apresentado valores abaixo dos preditos para a faixa etária avaliada, não foi possível estabelecer uma correlação entre estas variáveis.

Diferentemente do resultado do TC6, pela Escala de Katz, todos os idosos foram considerados independentes para realização das atividades de vida diária. A prevalência de idosos institucionalizados independentes também foi relatada em outro estudo, o qual enfatiza que existe uma relação direta entre a qualidade de vida e a independência funcional para as atividades diárias (ESTRADA et al., 2011). Além disso, acredita-se que, com o avançar da idade, exista uma maior probabilidade de surgirem mais problemas de saúde, maior dependência na realização das atividades da vida diária, mais isolamento e solidão, implicando na capacidade funcional e na qualidade de vida (MARRA et al., 2007).

A qualidade de vida na velhice tem sido muito associada a questões como independência e autonomia. No início da velhice observa-se uma maior redução da qualidade de vida nos aspectos social e psicológico, já para as idades mais avançadas o impacto é maior na forma como os idosos percebem sua saúde ou a redução da autonomia (FARENZENA et al., 2007; FERNANDEZMARTINEZ et al., 2012). Outras situações que podem influenciar na qualidade de vida são saúde bucal e nível de educação (FONTANIVE et al., 2013), como também os fatores socioeconômicos, por oferecer suporte material para o bem estar do individuo, influenciando a qualidade de vida, independência econômica e estabilidade financeira (FARENZENA et al., 2007). Maus tratos familiares também influenciam na redução da qualidade de vida (ESTRADA et al., 2011).

A saúde é um dos maiores recursos para o desenvolvimento social, econômico e pessoal, assim como, para uma importante qualidade de vida (GALISTEU et al., 2006). Uma das maneiras de evitar que o processo de envelhecimento se desenvolva de uma forma mais célere é manter-se ocupado (ALMEIDA; RODRIGUES, 2008). Entre os idosos que praticam atividades de lazer observa-se uma maior qualidade de vida nos domínios social e psicológico (FARENZENA et al., 2007). A manutenção de relações sociais com amigos da mesma geração favorece o bem-estar psicológico e social dos idosos (NERI; SOMMERHALDER, 2001). Entretanto, nem sempre esta participação social é uma situação rotineiramente observada entre idosos institucionalizados. 
Este estudo limitou-se a uma amostra de idosos de Foz do Iguaçu, Paraná, e o número reduzido de sujeitos reduz o poder da análise estatística. Sugere-se a continuidade do estudo, porém de forma longitudinal, buscando identificar melhor os fatores que interferem na qualidade de vida e no estado funcional desses idosos.

\section{Considerações finais}

Para uma amostra de idosos institucionalizados de Foz do Iguaçu, PR, não foram observadas correlações entre a força muscular inspiratória e expiratória com a capacidade funcional e qualidade de vida.

\section{Referências}

ALMEIDA, A. J. P. S.; RODRIGUES, V. M. C. P. A qualidade de vida da pessoa idosa institucionalizada em lares. Revista Latino-Americana de Enfermagem, v. 16, n. 6, p. 1025-1031, 2008. crossef

AMERICAN THORACIC SOCIETY. ATS Statement: guidelines for the six-minute walk test. American Journal of Respiratory and Critical Care Medicine, v. 166, n. 1, p. 111-117, 2002. crossef

ANJOS, D. M. C.; ARAÚJO, I. L.; BARROS, V. M.; PEREIRA, D. A. G.; PEREIRA, D. S. Avaliação da capacidade funcional em idosos diabéticos. Fisioterapia e Pesquisa, v. 19, n. 1, p. 7378, 2012. crossef

ARAUJO, C. O.; MAKDISSE, M. R. P.; PERES, P. A. T.; TEBEXRENI, A. S.; RAMOS, L. R.; MATSUSHITA, A. M.; CARVALHO, A. C. Diferentes padronizações do teste de caminhada de seis minutos como método para mensuração da capacidade de exercício de idosos com e sem cardiopatia clinicamente evidente. Arquivos Brasileiros de Cardiologia, v. 86, n. 3, p. 198-205, 2006. Crossef

BRITTO, R. R.; VIEIRA, D. S. R.; RODRIGUES, J. M.; PRADO, L. F.; PEREIRA, V. F. Comparação do padrão respiratório entre adultos e idosos saudáveis. Revista Brasileira de Fisioteria, v. 9, n. 3, p. 281-287, 2005.

CADER, A. S.; VALE, R. G. S.; MONTERIO, N.; PEREIRA, F. F.; DANTAS, E. H. M. Comparação de Pimáx e da qualidade de vida entre idosas sedentárias, asiladas e praticantes de hidroginástica. Fitness \& Performance Journal, v. 5, n. 2, p. 101-108, 2006.

DOURADO, V. Z. Equações de referência para o teste de caminhada de seis minutos em indivíduos saudáveis. Arquivos Brasileiros de Cardiologia, v. 96, n. 6, p. 128-138, 2011. crossef

ENRIGHT, P. L.; KRONMAL, R. A.; MANOLIO, T. A.; SCHENKER, M. B.; HYATT, R. E. Respiratory muscle stength in the elderly. Correlates and reference values. Cardiovascular Health Study Research Group. American Journal of Respiratory and Critical Care Medicine, v. 149, n. 2, p. 430-438, 1994. rossef

ESTRADA, A.; CARDONA, D.; SEGURA, A.M.; CHAVARRIAGA, L. M.; ORDÓÑEZ, J.; OSORIO, J. J. Calidad de vida de los adultos mayores de Medellín. Biomédica, v. 31, n. 4, p. 492502, 2011.

FARENZENA, W. P.; ARGEMAN, I. L.; MORIGUCHI, E.; PORTUGUEZ, M. V. Qualidade de vida em grupo de idosos de Veranópolis. Revista Kairós Gerontologia, v. 10, n. 2, p. 225-243, 2007. 
FERNANDEZ-MARTINEZ, B.; PREITO-FLORES, M. E.; FORJAZ, M. J.; FERNÁNDEZMAYORALAS, G.; ROJO-PÉREZ, F.; MARTÍNEZ-MARTÍN, P. Self-perceived health status in older adults: regional and sociodemographic inequalities in Spain. Revista de Saúde Pública, v.46, n. 2, p. 310-319, 2012.

FLECK, M. P. A.; LOUZADA, S.; XAVIER, M.; CHACHMOVICH, E.; VIEIRA, G.; SANTOS, L.; PINZON, V. Aplicação do versão em português do instrumento abreviado de avaliação da qualidade de vida "WHOQOL-bref”. Revista de Saúde Pública, v. 34, n. 2, p. 178-183, 2000. rossef

FONTANIVE, V.; ABEGG, C.; TSAKOS, G.; OLIVEIRA, M. The association between clinical oral health and genral quality of life: a population-based study of individuals aged 50-74 in Southern Brazil. Community Dentistry and Oral Epidemiology, v. 41, n. 2, p. 154-162, 2013. Crossef

FREITAS, F. S.; IBIAPINA, C. C.; ALVIM, C. G.; BRITTO, R. R.; PARREIRA, V. F. Relação entre força de tosse e nível funcional em um grupo de idosos. Revista Brasileira de Fisioterapia, v. 14, n. 6 , p. 470-476, 2010. Crossef

GALISTEU, K. J.; FACUNDIM, S. D.; RIBEIRO, R. C. H. M.; SOLER, Z. A. S. G. Qualidade de vida de idosos de um grupo de convivência com a mensuração da escala de Flanagem. Arquivos de Ciências da Saúde, v. 13, n. 4, p. 209-214, 2006.

LINO, V. T. S.; PEREIRA, S. R. M.; CAMACHO, L. A. B.; RIBEIRO FILHO, S. T.; BUKSMAN, S. Adaptação transcultural da escala de independência em atividades de vida diária (Escala de Katz). Cadernos de Saúde Pública, v. 24, n. 1, p. 103-112, 2008. crossef

MARRA, T. A.; PEREIRA, L. S. M.; FARIA, C. D. C. M.; PEREIRA, D. S.; MARTINS, M. A. A.; TIRADO, M. G. A. Avaliação das atividades de vida diária de idosos com diferentes níveis de demência. Revista Brasileira de Fisioterapia, v. 11, n. 4, p. 267-273, 2007. Grossef

MAZO, G. Z.; SILVA, A. H.; GONÇALVES, L. T.; BENEDETTI, T. B.; CLAUDINO, R, BENETTI, M. Z. Aptidão física de idosos institucionalizados: um estudo interinstitucional. ConScientiae Saúde, v. 10, n. 3, p. 473-479, 2011.

MINCATO, P. C.; FREITAS, C. L. R. Qualidade de vida dos idosos residentes em instituições asilares da cidade de Caxias do Sul - RS. Revista Brasileira de Ciência do Envelhecimento Humano, v. 4, n. 1, p. 127-138, 2007.

MOTA, J.; RIBEIRO, J. L.; CARVALHO, J.; MATOS, M. G. Atividade física e qualidade de vida associada a saúde em idosos participantes e não parcitipantes em programas regulares de atividade física. Revista Brasileira de Educação Física e Esporte, v. 20, n. 3, p. 219-225, 2006.

NEDER, J. A.; ANDREONI, S.; LERARIO, M. C.; NERY, L. E. Reference values for lung function tests. II. Maximal respiratory pressures and voluntary ventilation. Brazilian Journal of Medical and Biological Research, v. 32, n. 6, p. 719-727, 1999.

NERI, A. L.; SOMMERHALDER, C. As várias faces do cuidado e do bem-estar do cuidador. In: NERI, A. L. Cuidar de idosos no contexto da família: questões psicológicas e sociais. Campinas: Átomo e Alínea, 2001. 
PAVAN, K.; LETKASKE, F.; SAKAMOTO, T.; CARVALHO, M. M.; MARANGONI, B. E. M.; NISHINO, L. K.; LIANZA, S. Reabilitação vestibular em pacientes neurológicos. Medicina de Reabilitação, v. 2, n. 2, p. 31-36, 2010.

PETTENON, R.; MILANO, D.; BITTENCOURT, D. C.; SHNEIDER, R. H. Adaptação funcional do aparelho respiratório e da postura do idoso. Revista Brasileira de Ciência do Envelhecimento Humano, v. 5, n. 2, p. 64-77, 2008.

SIMÕES, L. A.; DIAS, J. M. D.; MARINHO, K. C.; PINTO, C. L. L. R.; BRITTO, R. R. Relação da função muscular respiratória e de membros inferiores de idosos comunitários com a capacidade funcional avaliada por teste caminhada. Revista Brasileira de Fisioteria, v. 14, n. 1, p. 24-30, 2010. rossef

VASCONCELOS, J. A. C.; BRITTO, R. R.; PARREIRA, V. F.; CURY, A. C.; RAMIRO, S. M. Pressões respiratórias máximas e capacidade funcional em idosas assintomáticas. Fisioterapia em Movimento, v. 20, n. 3, p. 93-100, 2007. 